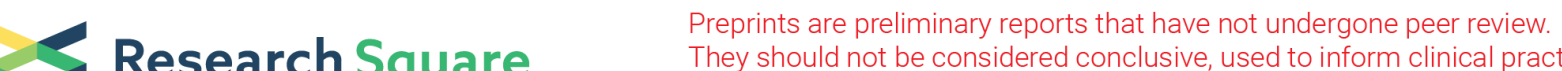 \\ They should not be considered conclusive, used to inform clinical practice, or referenced by the media as validated information.
}

\section{NETs Enhance STING To Promote Surgical Adhesion}

Qiongyu Hu

Nanjing Drum Tower Hospital: Nanjing University Medical School Affiliated Nanjing Drum Tower Hospital

\section{Xiaofei Shen}

Nanjing Drum Tower Hospital: Nanjing University Medical School Affiliated Nanjing Drum Tower Hospital

\section{Meng Wang}

Nanjing Drum Tower Hospital: Nanjing University Medical School Affiliated Nanjing Drum Tower Hospital

\section{Xiaofeng Lu}

Nanjing Drum Tower Hospital: Nanjing University Medical School Affiliated Nanjing Drum Tower Hospital

\section{Song Liu}

Nanjing Drum Tower Hospital: Nanjing University Medical School Affiliated Nanjing Drum Tower Hospital

Wenxian Guan ( $\square$ guan_wenxian@sina.com )

Nanjing Drum Tower Hospital https://orcid.org/0000-0002-2171-6265

\section{Research}

Keywords: Postoperative adhesion, NETs, STING

Posted Date: June 7th, 2021

DOI: https://doi.org/10.21203/rs.3.rs-515654/v1

License: (c) (i) This work is licensed under a Creative Commons Attribution 4.0 International License. Read Full License 


\section{Abstract \\ Background}

Postoperative adhesion (PA) following abdominal surgery may cause bowel obstruction, chronic pain, infertility, or even death. Knowledge of adhesion biology is limited, and preventive agents in clinical trials have failed to achieve efficacy.

\section{Results}

In the present study, we showed that neutrophils accumulate in the injured peritoneum at early stage of $\mathrm{PA}$, and neutrophils within the ischemic buttons undergo cell death and form neutrophil extracellular traps (NETs) that contribute to PA. Neutrophil depletion reduces adhesion burden at 7 days after adhesion induction. Peptidylarginine deiminase 4 (PAD4), an essential enzyme for NET formation, is increased in ischemic buttons. Degradation of NETs by DNase 1 and suppression of NET formation by pharmacologic inhibition of PAD4 alleviated adhesion burden, collogen deposition and fibrosis formation. Mechanistically, administration of DNase I and PAD inhibitor reduces STING-mediated inflammatory response. STING deficiency attenuates adhesion burden, collogen deposition, and a-SMA production in the adhesive tissues at 7 days after surgery.

\section{Conclusions}

Collectively, our findings reveal NETs/STING signaling as a therapeutic target to prevent PA.

\section{Introduction}

Postoperative adhesion (PA) is the most common complication of abdominal surgery, which can lead to intestinal obstruction, abdominal pain, female infertility, and even death [1]. Repeated surgery including adhesiolysis can cause large burden of morbidity [2]. However, effective strategy is unavailable to prevent or treat PA [3]. Deep understanding of molecular mechanisms of PA process allows the design of new anti-adhesion treatment.

Neutrophils are recognized as the earliest leukocytes in the response to tissue damage [4]. Adhesion tissue was obtained from adhesive small bowel obstruction (ASBO) patients, and pathological staining indicated that infiltrating neutrophils within thick peritoneum was accompanied by increased DNA fibers structure (Supplementary Fig. 1), which indicated NETs (neutrophil extracellular traps, NETs) formation. NETs contain double-stranded DNA, histone, and granule proteins including neutrophil elastase, cathepsin G, and myeloperoxidase (MPO) [5]. These NETs were associated with immune disorder, inflammation, and fibrosis [6]. However, whether NETs contribute to favorable or poor outcomes during PA formation remains unclear. A recent study found that delayed NETs formation was showed in the adhesive tissue in 
mouse [7]; However the underlying mechanism in NETs formation and their cascade effect remain largely unknown during PA development.

Mechanism during later stage of PA formation involving fibrin deposition and fibrinolysis has been wellstudied; In contrast, the cellular and molecular detail during initial stage requires extensive elucidation [8]. In this study, we discover early neutrophil-dependent NETs formation in injured peritoneum in a murine PA model. Importantly, depletion of neutrophils or disruption of NETs formation improves surgical traumainduced early inflammatory response, collagen formation, and subsequent fibrosis. Furthermore, either neutrophil depletion or PAD4 inhibitor suppresses PA-mediated upregulation of the DNA sensor cyclic stimulator of interferon genes (STING). Collectively, our findings demonstrated that NETs/STING pathway could become a promising target for the prevention of PA clinically.

\section{Methods}

\section{ASBO patients}

Our study was performed in accordance to the Recommendations of Guidelines for Clinical Trials by the Ethics Committee of Nanjing Drum Tower Hospital. Adhesive peritoneum tissues were obtained from ASBO patients during laparotomy or laparoscopy (Supplementary Fig.1a). Informed consent was obtained from all subjects after surgery.

\section{Adhesion induction}

Adhesion-induction surgery was performed as previously described [8]. In brief, a small portion of the peritoneum was grasped and ligated at its base using 4-0 silk suture (Ethicon), creating a standardized peritoneal button. This was repeated for a total of four buttons (one in each quadrant). Light abrasion on each button and on the adjacent liver, cecum, and intestine was performed using surgical brash. Finally, peritoneum and skin were closed using 4-0 silk suture. Mouse ischemic button model is easily reproducible to induce intra-abdominal adhesions compared to other models as previously demonstrated [9]. The experimental procedure in this study was approved by the Animal Care and Use Committee of Nanjing Drum Tower Hospital.

\section{Histology, IHC and immunofluorescence}

Tissues were fixed in $2 \%$ PFA overnight at $4^{\circ} \mathrm{C}$ and were embedded in paraffin. paraffin sections were cut at $5 \mu \mathrm{m}$. H\&E, Masson's trichome stains, and immunohistochemistry (IHC) were performed following standard protocols.

For IHC staining, slides were incubated with anti-APD4 antibody, anti-citH3 antibody, and anti-a-SMA antibodies according to the manufacture's instruction. Nuclei were stained with DAPI (Invitrogen).

Immunofluorescence experiment was performed on paraffin sections. NETs associated proteins were evaluated by immunofluorescent staining using rabbit mouse anti-mouse MPO, and rabbit anti-mouse 
citH3 (Abcam, Cambridge, UK). DAPI was use to counterstain DNA. Images were acquired using a Leica DMI 4000 B fluorescence microscope.

\section{ELISA assay}

Enzyme-linked immunosorbent assays (ELISA) was performed to evaluate the production of inflammatory cytokines, including IL-1 $\beta, \mathrm{IL}-6$, and TNF-a according to the manufacturer's instructions.

\section{Neutrophil depletion}

Mice were injected with $100 \mu \mathrm{g}$ intraperitoneal (i.p.) monoclonal anti-mouse Ly6G at 12h or 3d after induction of ischemic button. Rat lgG2a isotype control was administered in the same way. To verify neutrophil depletion, blood neutrophil levels were evaluated by flow cytometry and analyzed using FlowJo V10 software as previously described [10].

\section{DNase I and PAD inhibitor administration}

Mice received i.p. injection of $50 \mu \mathrm{g}$ DNase $\mathrm{I}$ at $2 \mathrm{~h}, 12 \mathrm{~h}$ or $3 \mathrm{~d}$ after induction of ischemic button. Control counterparts were injected with PBS. PAD inhibitor Cl-amidine was dissolved in dimethyl sulfoxide (DMSO) as previously described. The stock solution was dissolved in saline and injected i.p. (10 mg/kg) at $2 \mathrm{~h}, 12 \mathrm{~h}$ and $3 \mathrm{~d}$ after adhesion induction. Vehicle (DMSO) was administered as control (Fig.3a).

\section{Western blot}

The peritoneal ischemic buttons were dissected after the mice were sacrificed, and the protein from adhesive tissues and HPMCs were extracted using RIPA lysis buffer. Proteins were separated by $10 \%$ SDS-PAGE, and transferred to PVDF membrane. After blocking with 5\% milk, the membranes were incubated with anti-Ly6G, anti-PAD4, anti-citH3, anti-p-TBK1 and anti-p-IRF3 at $4{ }^{\circ} \mathrm{C}$ overnight. GAPDH served as internal control.

\section{Statistical analysis}

All statistical analyses were evaluated using SPSS 19.0 and GraphPad Prism 6. All data were presented as mean \pm SEM. Unpaired t test and one-way analysis were used to compare continuous variables between groups. $\mathrm{P}<0.05$ was considered significant.

\section{Results}

\section{Early neutrophil infiltration causes peritoneal structure damage in murine PA}

Mice were subjected to ischemic button and analyzed at $6 \mathrm{~h}, 12 \mathrm{~h}$ and $24 \mathrm{~h}$. The damaged peritoneum was markedly thickened and showed collagen deposition by H\&E and Masson staining (Fig. 1a). Increased polymorphonuclear leukocyte were observed in the buttons compared with sham group. MPO staining showed that neutrophils were visualized within thicken peritoneum after adhesion induction (Fig.1b). A 5- 
fold increase in total content of the neutrophil enzyme MPO was found in the buttons at 24h after surgical trauma (Fig.1c). Using Ly6G antibody, the levels of neutrophil continued to increase within $24 \mathrm{~h}$ after adhesion induction (Fig.1d).

To investigate the role of neutrophils in fibrosis development during delayed phases of PA, we depleted neutrophil using anti-Ly6G antibody. Peripheral white blood cell counts and neutrophils were significantly decreased by anti-Ly6G treatment (Supplementary Fig. 2a,2b). Correspondingly, H\&E and immunostaining showed that neutrophil recruitment in the ischemic buttons was significantly reduced in anti-Ly6G-treated mice compared to IgG-treated mice (Supplementary Fig. 2c,2d). Mice receiving anti-Ly6G antibody after injury exhibited a significantly decreased adhesion burden compared with controls (Supplementary Fig.2e).

\section{PA induces early NETs formation}

We subsequently elucidate whether NETs formation occurs in ischemic buttons and affects adhesion outcomes. IHC and Western blot analysis showed an increased production of PAD4 and citH3 within the ischemic buttons at 24h, indicating that NETs may play a vital role at the early stage of PA (Fig.2a, 2b). Microscopic analysis detected positive staining of citH3 and MPO, overlapping with diffused DNA scaffolds, that indicated increased formation of NETs structure in the buttons (Fig.2c). Interestingly, colocalization of citH3 and MPO was not observed at $6 \mathrm{~h}$ but appeared at $12 \mathrm{~h}$ following adhesion induction (Supplementary Fig.3a).

\section{Disruption of NETs structure and inhibition of NETs formation alleviate adhesion formation}

We next investigate whether disruption of NETs could alleviated PA formation in mice. PAD4 acts as a driver of NETs formation. Treatment with DNase I and Cl-amidine (PAD4 inhibition) failed to affect the amount of neutrophils in the ischemic buttons (Supplementary Fig. 4a, 4b) but significantly reduced citH3 levels confirmed by IHC staining (Fig. 3b). Immunofluorescence staining for $\mathrm{CiH} 3$ and MPO in ischemic buttons at $24 \mathrm{~h}$ found that administration of either DNase I or Cl-amidine could significantly prevent NETs formation in injured peritoneum. In addition, adhesion burden, collogen deposition and fibrosis formation were significantly reduced following DNase I and Cl-amidine treatment after 7 days compared with controls (Supplementary Fig.4c and Fig.3d).

\section{NETs are responsible for STING-mediated PA formation}

An increased production of inflammatory mediators in the early stage leads to extracellular matrix formation during PA, and NETs are associated with increased inflammation [11]. We next evaluated whether NETs regulate inflammatory response. Inflammatory mediators (IL-1 $\beta$, IL- 6 and TNF- $\alpha$ ) were significantly suppressed in the ischemic buttons following DNase I treatment at 24h after surgery (Supplementary Fig.5a).

Free DNA binds with cyclic GMP-AMP synthase to promote STING-dependent inflammatory response [12]. We detected active STING signaling and phosphorylated TANK-binding kinase 1 ( $\mathrm{pTBK} 1$ ) and TBK1- 
dependent IFN regulatory factor 3 (IRF3) activation at 24h after adhesion induction (Fig.4a). Neutrophil depletion by anti-Ly6G inhibited STING signaling in the ischemic buttons (Fig.4b). Furthermore, PAD4 inhibitor also suppressed STING-related molecules including PTBK1 and pIRF3 compared with controls (Fig.4c).

We next investigated whether STING-deficient mice were more resistant to PA formation. Adhesion burden, collogen deposition, and a-SMA production were significantly decreased in the adhesive tissues at 7d in STING-deficient mice after surgery (Fig.4e and Supplementary Fig.6a). Interestingly, we unexpectedly found that STING deficiency was able to reduce NETs formation within the ischemic buttons at 24h, suggesting a contribution of STING signaling to NETosis (Supplementary Fig.7a).

\section{Discussion}

PA develops in most patients receiving abdominal surgery. Despite its clinical importance, cellular and molecular processes and preventive strategy of PA remains largely unknown [9]. Previous studies suggested that neutrophil chemotaxis played an important role in exacerbating adhesion[8, 13]. A recent study suggested that delayed NETs formation occurred in adhesive tissues surface[7]. In our study, early neutrophil infiltration and NETs formation were showed in injured peritoneum. Collectively, neutrophil accumulation and NETs formation are essential for both early and late stages of PA formation.

Neutrophils are important in contributing to the transition from pro-inflammatory response to pro-fibrotic condition [14]. Our recent study showed accumulation of neutrophils in adhesive tissues during all stages of adhesion, and the mount of neutrophils reached to the peak point within 1-2 days [4]. Therefore, understanding the molecular function of neutrophils may help explain the formation of adhesions. Indeed, neutrophil depletion reduced adhesion burden at 7 days after adhesion induction. Reduction in circulating neutrophils could possibly lead to immune compromise state during peri-operative period. Further studies towards the consequence of neutrophil depletion on side effects after surgery will be essential.

Neutrophils release NETs-associated cytotoxic proteases such as histone, elastase and MPO, which was associated with inflammatory response, collagen production ECM deposition [14, 15]. NETs could also promote differentiation and function of fibroblasts, that contribute to organ fibrosis [15, 16]. Our data suggested that activated neutrophils release excessive NETs at injured peritoneum. Digestion of NETs with DNase I significantly decreased inflammatory response, collagen production and fibrosis formation. These findings strongly indicated that NETs could be critical for neutrophil-dependent inflammation and pro-fibrotic condition during early and late stage of PA process.

PAD4 is an essential enzyme for NETs formation [17]. We demonstred that PAD4 was significantly increased in the ischemic buttons after surgery. Consistently, we found that PAD4 inhibition suppressed early NETs formation, and subsequently alleviated collagen deposition, fibrosis formation, and adhesion burden. These data suggest that PAD4 depletion could ameliorate fibrosis development. 
STING is an vital regulator of tissue inflammation and fibrosis in multiple organ, including lung [18], liver [19], kidney [20] and heart [21]. Our study suggested that PA activates the DNA sensor STING and induces the production of inflammatory cytokines. STING depletion in mice decreased collogen deposition and aSMA production. In addition, degradation and inhibition of NETs suppressed the activation of STING signaling. Recent studies also highlight that NETs are responsible for STING activation during inflammatory or immune diseases [11,22]. Therefore, STING-mediated inflammatory response may link NETs and PA formation. Interestingly, we found that depletion of STING in turn inhibited the formation of NETs structure. A recent study also suggested that STING pathway may contribute to the induction of NETs by mtDNA stimulation [23]. Therefore, it possible that NETs/STING pathway may form a vicious cycle during early stage of adhesion, which eventually contribute to fibrosis and PA formation.

\section{Conclusion}

Our study revealed that early neutrophils recruited in injured peritoneum that further released NETs structure, and subsequently activated STING-associated inflammatory response. Furthermore, we demonstrated that disruption of NETs and inhibition of STING signaling could significantly decrease the adhesion burden. Our data suggested that NETs/STING pathways could serve as targets for PA prevention.

\section{Declarations}

\section{*Ethics approval and consent to participate}

Our study was performed in accordance to the Recommendations of Guidelines for Clinical Trials by the Ethics Committee of Nanjing Drum Tower Hospital. The experimental procedure in this study was approved by the Animal Care and Use Committee of Nanjing Drum Tower Hospital.

\section{*Consent for publication}

Not applicable.

\section{*Availability of data and material}

Not applicable.

\section{*Competing interests}

All authors declare no conflicts of interest.

\section{*Funding}

This work was supported by the National Natural Science Foundation of China (81602103); Distinguished Young Scholar Project of Medical Science and Technology Development Foundation of 
Nanjing Department of Health (JQX17005). Natural Science Foundation of Jiangsu Province (BK20200052).

\section{*Authors' contributions}

WG and SL conceptualized the study. QH wrote the original draft. LS, XS, MW and QH contributed to writing, reviewing, and editing the article. SL contributed in acquiring funds. WG supervised the study.

\section{*Acknowledgements}

Not applicable

\section{References}

1. Krielen P, Stommel MWJ, Pargmae P, Bouvy ND, Bakkum EA, Ellis H, Parker MC, Griffiths EA, van Goor $\mathrm{H}$, Ten Broek RPG: Adhesion-related readmissions after open and laparoscopic surgery: a retrospective cohort study (SCAR update). Lancet (London, England) 2020, 395(10217):33-41.

2. ten Broek RP, Strik C, Issa Y, Bleichrodt RP, van Goor H: Adhesiolysis-related morbidity in abdominal surgery. Annals of surgery 2013, 258(1):98-106.

3. Fischer A, Koopmans T, Ramesh P, Christ S, Strunz M, Wannemacher J, Aichler M, Feuchtinger A, Walch $A$, Ansari $M$ et al: Post-surgical adhesions are triggered by calcium-dependent membrane bridges between mesothelial surfaces. Nature communications 2020, 11(1):3068.

4. Hu Q, Xia X, Kang X, Song P, Liu Z, Wang M, Lu X, Guan W, Liu S: A review of physiological and cellular mechanisms underlying fibrotic postoperative adhesion. International journal of biological sciences 2021, 17(1):298-306.

5. Tan C, Aziz M, Wang P: The vitals of NETs. Journal of leukocyte biology 2020.

6. Fine N, Tasevski N, McCulloch CA, Tenenbaum HC, Glogauer M: The Neutrophil: Constant Defender and First Responder. Frontiers in immunology 2020, 11:571085.

7. Tsai JM, Shoham M, Fernhoff NB, George BM, Marjon KD, McCracken MN, Kao KS, Sinha R, Volkmer AK, Miyanishi $M$ et al: Neutrophil and monocyte kinetics play critical roles in mouse peritoneal adhesion formation. Blood advances 2019, 3(18):2713-2721.

8. Tsai JM, Sinha R, Seita J, Fernhoff N, Christ S, Koopmans T, Krampitz GW, McKenna KM, Xing L, Sandholzer $\mathrm{M}$ et al: Surgical adhesions in mice are derived from mesothelial cells and can be targeted by antibodies against mesothelial markers. Science translational medicine 2018, 10(469).

9. Ito T, Shintani Y, Fields L, Shiraishi M, Podaru MN, Kainuma S, Yamashita K, Kobayashi K, Perretti M, Lewis-McDougall $\mathrm{F}$ et al: Cell barrier function of resident peritoneal macrophages in post-operative adhesions. Nature communications 2021, 12(1):2232.

10. Kang L, Yu H, Yang X, Zhu Y, Bai X, Wang R, Cao Y, Xu H, Luo H, Lu L et al: Neutrophil extracellular traps released by neutrophils impair revascularization and vascular remodeling after stroke. Nature communications 2020, 11(1):2488. 
11. Wang R, Zhu Y, Liu Z, Chang L, Bai X, Kang L, Cao Y, Yang X, Yu H, Shi MJ et al: Neutrophil extracellular traps promote tPA-induced brain hemorrhage via cGAS in mice with stroke. Blood 2021.

12. Decout A, Katz JD, Venkatraman S, Ablasser A: The cGAS-STING pathway as a therapeutic target in inflammatory diseases. Nature reviews Immunology 2021:1-22.

13. Uyama N, Tsutsui H, Wu S, Yasuda K, Hatano E, Qin XY, Kojima S, Fujimoto J: Anti-interleukin-6 receptor antibody treatment ameliorates postoperative adhesion formation. Scientific reports 2019, $9(1): 17558$.

14. Zhou P, Li T, Jin J, Liu Y, Li B, Sun Q, Tian J, Zhao H, Liu Z, Ma S et al: Interactions between neutrophil extracellular traps and activated platelets enhance procoagulant activity in acute stroke patients with ICA occlusion. EBioMedicine 2020, 53:102671.

15. Suzuki M, Ikari J, Anazawa R, Tanaka N, Katsumata Y, Shimada A, Suzuki E, Tatsumi K: PAD4 Deficiency Improves Bleomycin-induced Neutrophil Extracellular Traps and Fibrosis in Mouse Lung. American journal of respiratory cell and molecular biology 2020, 63(6):806-818.

16. Chrysanthopoulou A, Mitroulis I, Apostolidou E, Arelaki S, Mikroulis D, Konstantinidis T, Sivridis E, Koffa M, Giatromanolaki A, Boumpas DT et al: Neutrophil extracellular traps promote differentiation and function of fibroblasts. The Journal of pathology 2014, 233(3):294-307.

17. Hu Q, Ren H, Hong Z, Wang C, Zheng T, Ren Y, Chen K, Liu S, Wang G, Gu G et al: Early Enteral Nutrition Preserves Intestinal Barrier Function through Reducing the Formation of Neutrophil Extracellular Traps (NETs) in Critically III Surgical Patients. Oxidative medicine and cellular longevity 2020, 2020:8815655.

18. Chung KW, Dhillon P, Huang S, Sheng X, Shrestha R, Qiu C, Kaufman BA, Park J, Pei L, Baur J et al: Mitochondrial Damage and Activation of the STING Pathway Lead to Renal Inflammation and Fibrosis. Cell metabolism 2019, 30(4):784-799.e785.

19. Luo X, Li H, Ma L, Zhou J, Guo X, Woo SL, Pei Y, Knight LR, Deveau M, Chen Y et al: Expression of STING Is Increased in Liver Tissues From Patients With NAFLD and Promotes Macrophage-Mediated Hepatic Inflammation and Fibrosis in Mice. Gastroenterology 2018, 155(6):1971-1984.e1974.

20. Allison SJ: STING activation by cytoplasmic mtDNA triggers renal inflammation and fibrosis. Nature reviews Nephrology 2019, 15(11):661.

21. Zhang Y, Chen W, Wang Y: STING is an essential regulator of heart inflammation and fibrosis in mice with pathological cardiac hypertrophy via endoplasmic reticulum (ER) stress. Biomedicine \& pharmacotherapy = Biomedecine \& pharmacotherapie 2020, 125:110022.

22. Apel F, Andreeva L, Knackstedt LS, Streeck R, Frese CK, Goosmann C, Hopfner KP, Zychlinsky A: The cytosolic DNA sensor cGAS recognizes neutrophil extracellular traps. Science signaling 2021, 14(673).

23. Liu L, Mao Y, Xu B, Zhang X, Fang C, Ma Y, Men K, Qi X, Yi T, Wei Y et al: Induction of neutrophil extracellular traps during tissue injury: Involvement of STING and Toll-like receptor 9 pathways. Cell proliferation 2020, 53(10):e12775. 


\section{Figures}

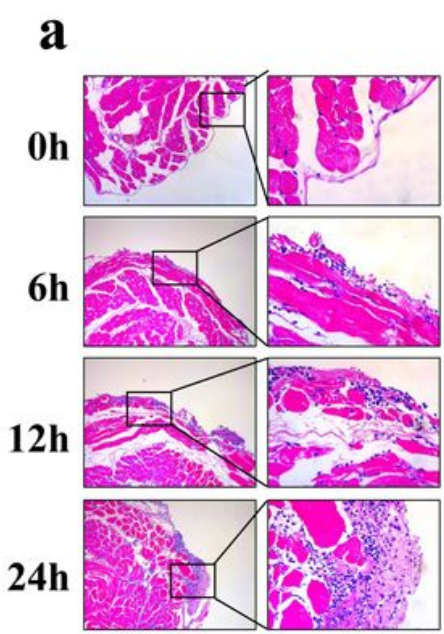

HE

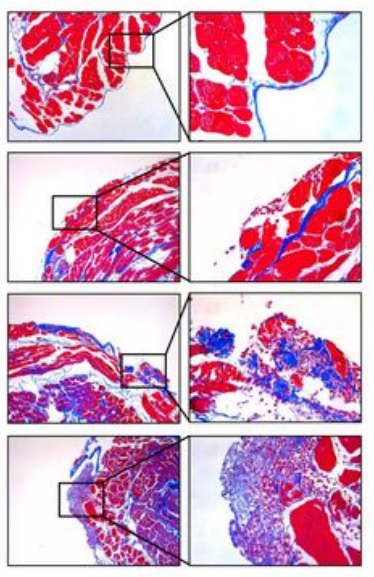

Masson b
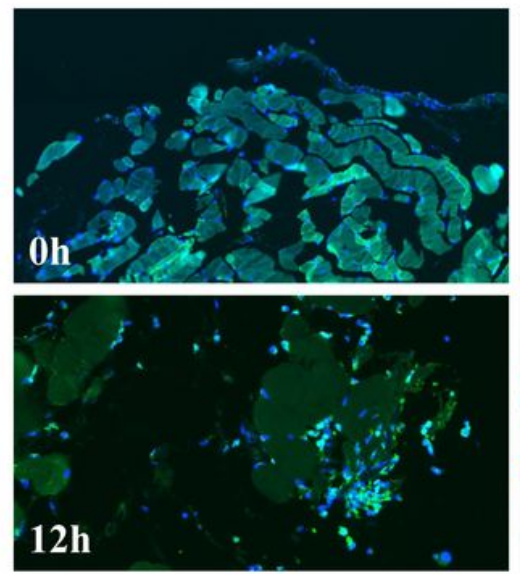
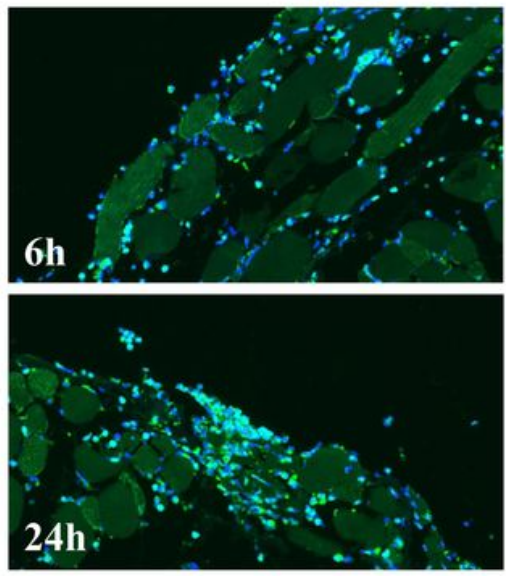

MPO

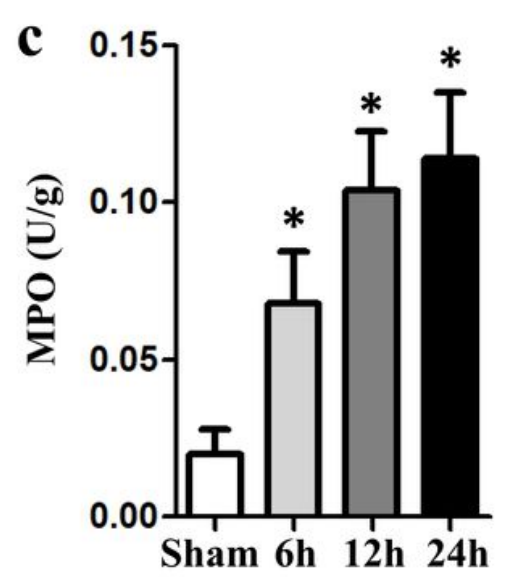

d
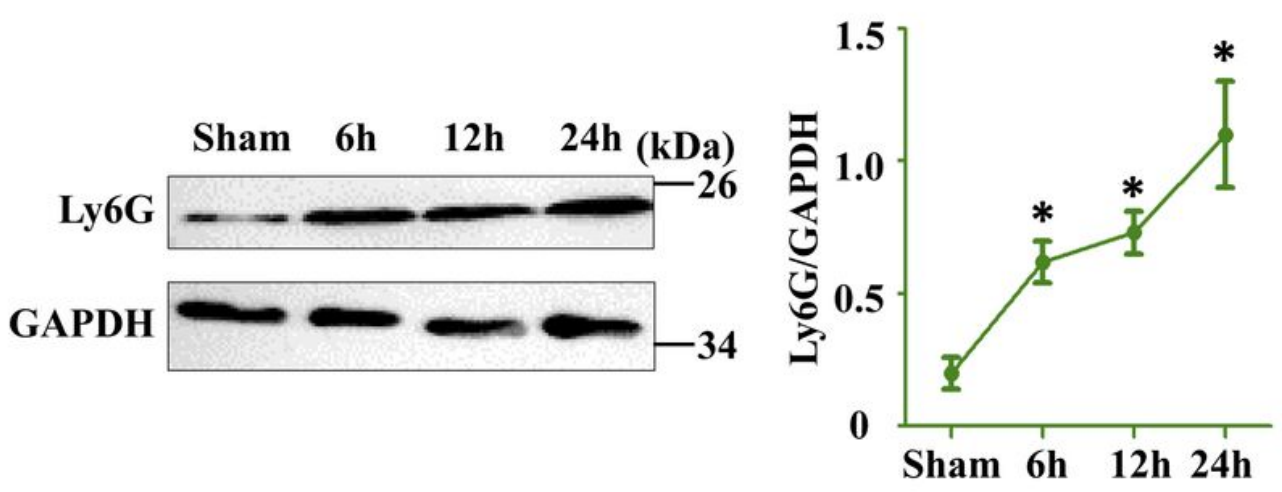

Figure 1

Neutrophil influx occurred in the early stage of adhesion formation. (a) Images show H\&E and Masson staining of ischemic buttons at 30 min and 1, 2, and 4 hours after button induction; (b) microscopy images of MPO staining in the ischemic buttons; (c) Quantification of MPO activity in the buttons of peritoneum at 24 hours after surgery; (d) Representative immunoblots of the time course of neutrophils appearance. ${ }^{*} p<0.05$. Data are presented as mean \pm SD. 

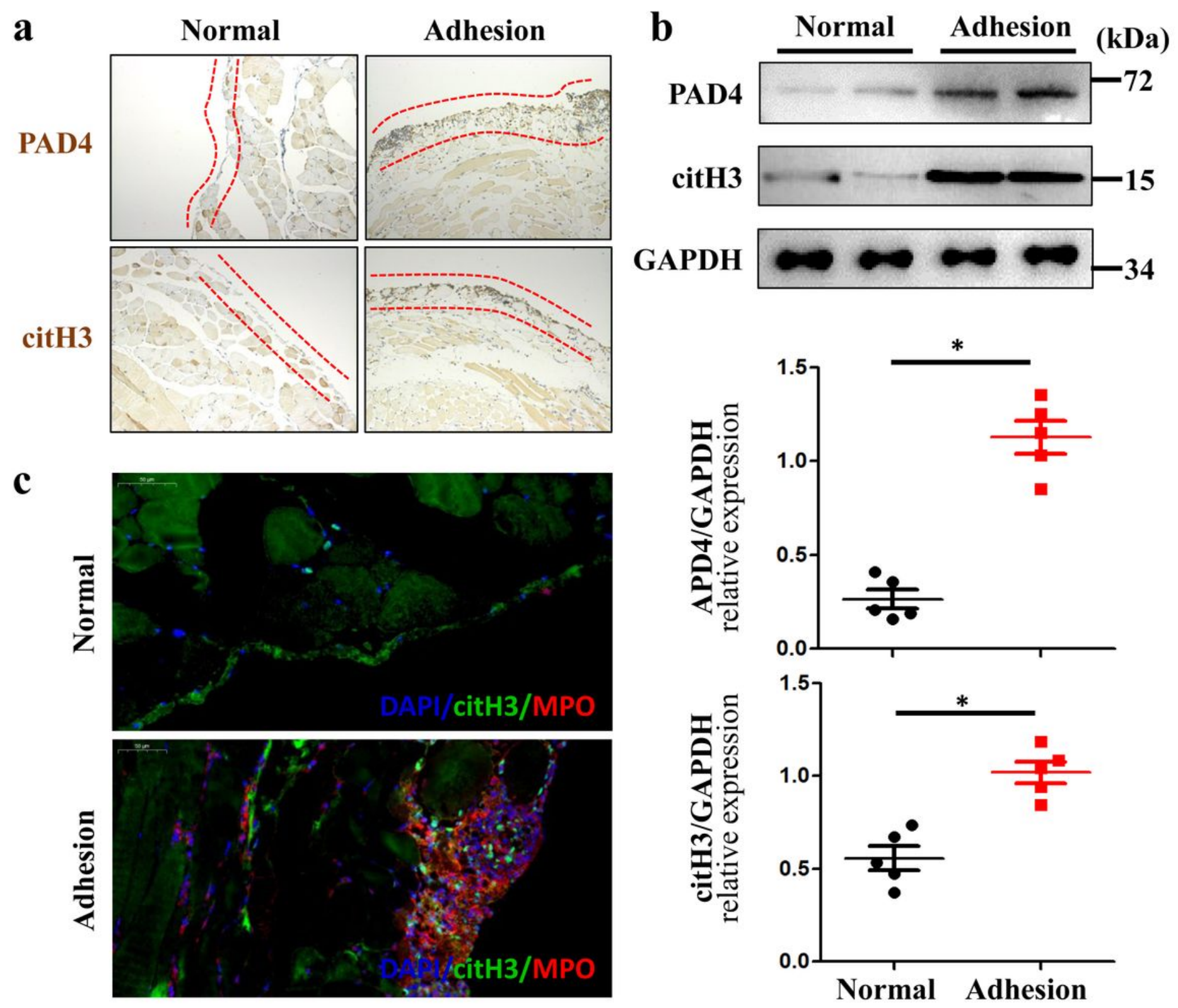

Figure 2

Surgical trauma induces early NETs formation after induction of ischemic button. (a) Representative images of PAD4 and citH3 by IHC staining at 24 hours; (b) quantification of the PAD4 and citH3 levels by Western blot; (c) Representative confocal images showing NET formation in the ischemic button of mice at 24 hours after surgery. ${ }^{*} p<0.05$. Data are presented as mean $\pm S D$. 
a

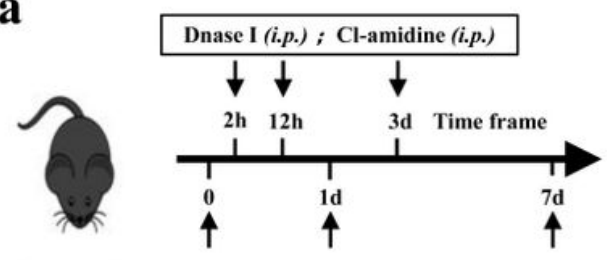

C57BL/6 IB induction

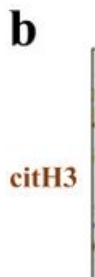

Harvest and analysis

c

Adhesion (24h)

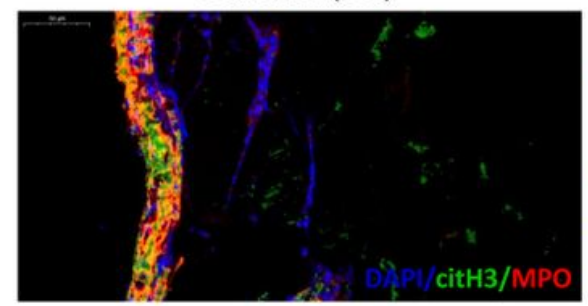

d
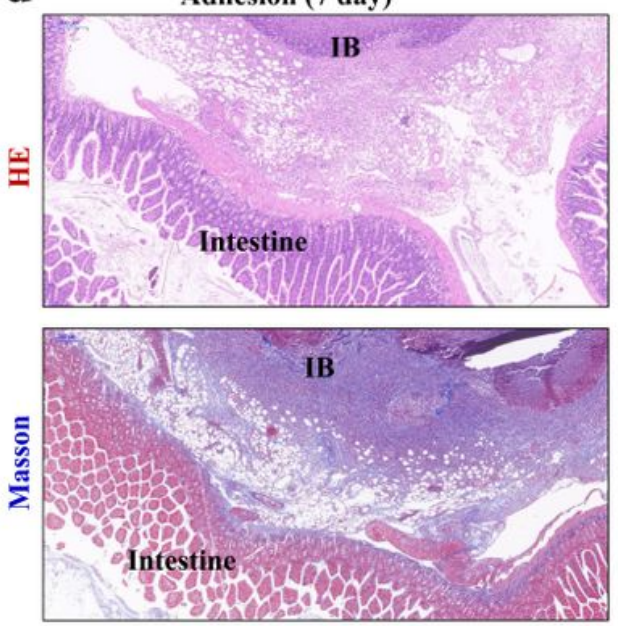
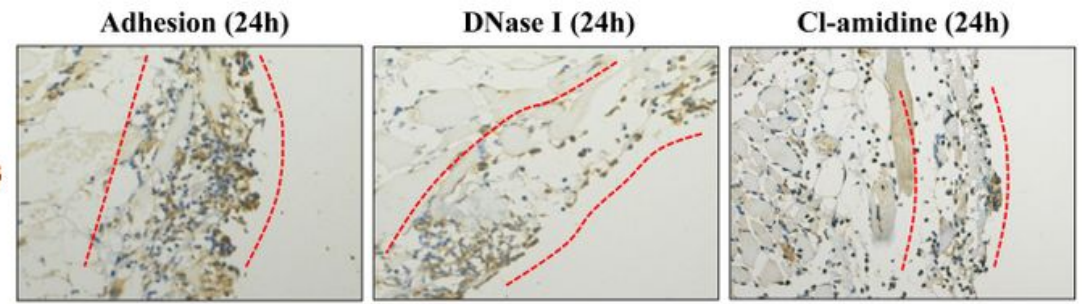

DNase I (24h)

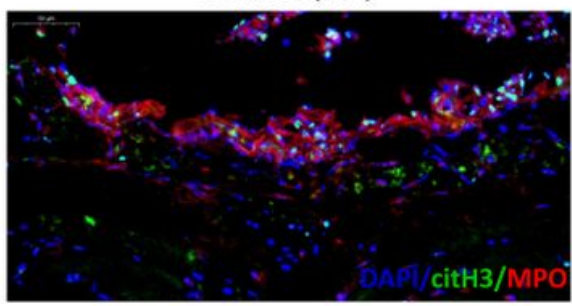

DNase I (7 day)
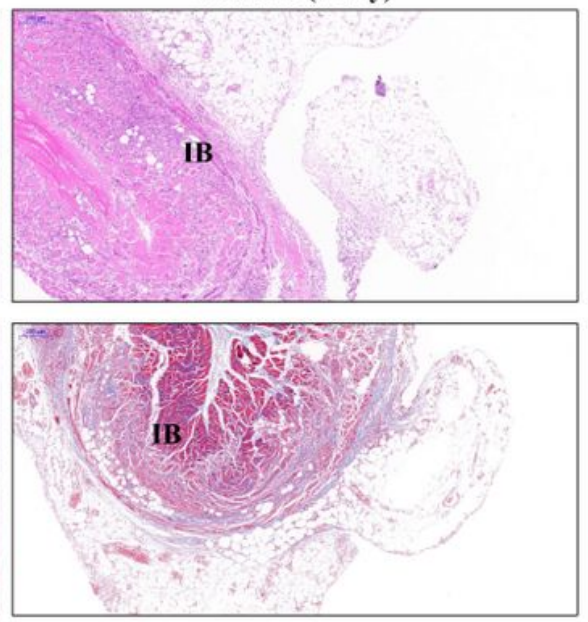

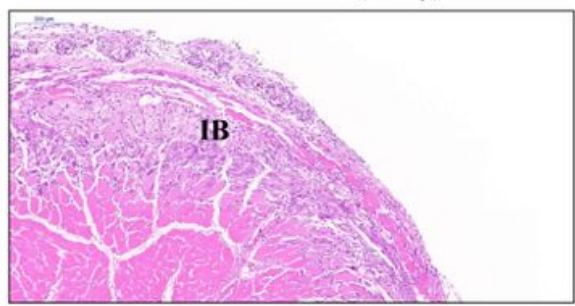

Cl-amidine (24h)

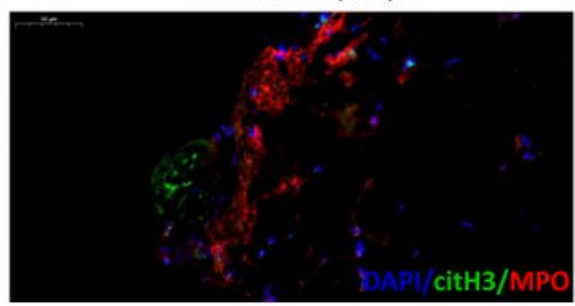

Cl-amidine (7 day)

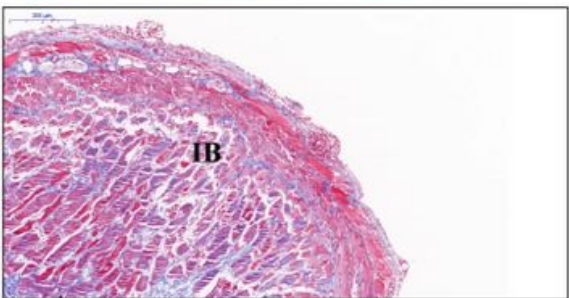

Figure 3

Inhibition of NETs formation affects adhesion stability after induction of ischemic button. (a) Time frame of DNase I and Cl-amidine administration; quantification of citH3 levels (b) and NETs formation (c) within the ischemic buttons at 24 hours in mice treated with vehicle or DNase 1 or Cl-amidine; (d) H\&E and Masson staining of mice adhesive tissue 7 days after button induction and treatment with DNase I or $\mathrm{Cl}$ amidine. 

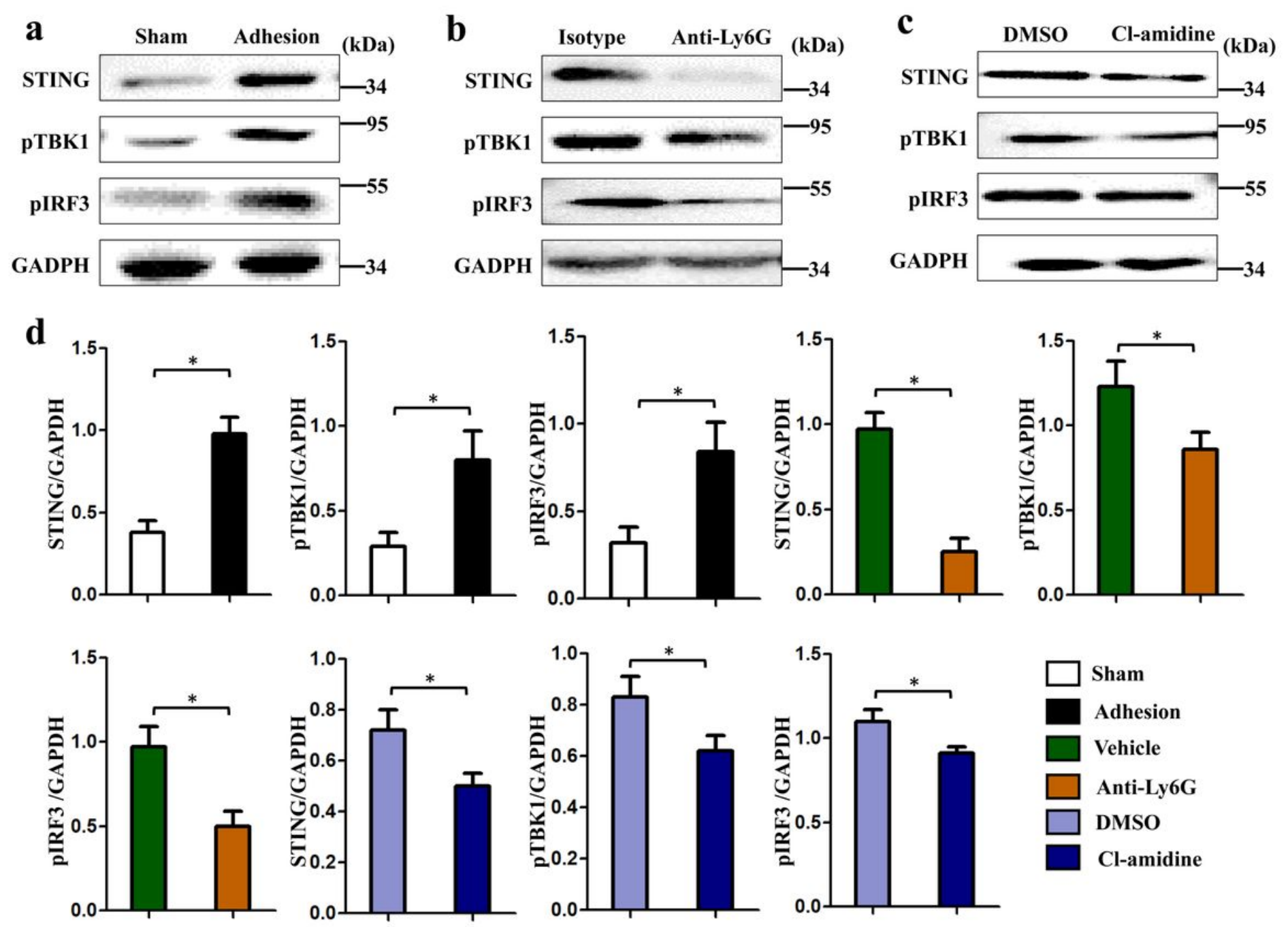

e
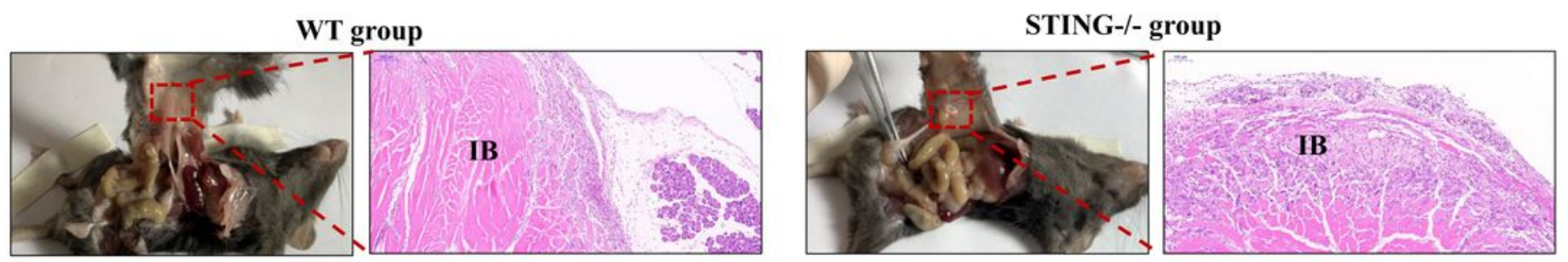

Figure 4

NETs are responsible for STING-mediated adhesion formation. (a) Immunoblot analysis of STING, pTBK1, and pIRF3 in the ischemic buttons 24 hours after surgery, and treatment with anti-Ly6G antibody (b) or Cl-amidine (c). H\&E staining of adhesive tissue between WT mice and STING-/- mice.

\section{Supplementary Files}

This is a list of supplementary files associated with this preprint. Click to download.

- SupplementaryFigure1.jpg 
- SupplementaryFigure2.jpg

- SupplementaryFigure3.jpg

- SupplementaryFigure4.jpg

- SupplementaryFigure5.jpg

- SupplementaryFigure6.jpg

- SupplementaryFigure7.jpg 